\title{
Pacific
}

Journal of

Mathematics

\section{SOME REMARKS ABOUT CLOSED CONVEX CURVES}

Ke Ou and Shengliang Pan 


\title{
SOME REMARKS ABOUT CLOSED CONVEX CURVES
}

\author{
Ke OU AND Shengliang Pan
}

\begin{abstract}
We introduce a function $w_{k}(\theta)$ for closed convex plane curves, and then prove a geometric inequality involving $w_{k}(\theta)$ and the area $A$ enclosed by the curve. As a by-product, we give a new proof of the classical isoperimetric inequality. Finally, we give some properties of convex curves with $w_{k}(\theta)$ being constant and pose an open problem motivated by the elegant Blaschke-Lebesgue theorem.
\end{abstract}

\section{Introduction}

Geometric inequalities involving convex sets have received much attention during the last centuries; see for example [Bonnesen and Fenchel 1934; Burago and Zalgaller 1988; Schneider 1993]. Among them the isoperimetric inequalities are of special interest; see [Ball 1991; Blaschke 1956; Bonnesen 1929; Osserman 1978; 1979; Pan and Zhang 2007; Schneider 1993] and references therein. For convex curves in the Euclidean plane $\mathbb{R}^{2}$, there are also many interesting inequalities involving their geometric quantities such as inradius, outradius, width, area, length and curvature or radius of curvature; see for example [Chernoff 1969; Gage 1983; Green and Osher 1999; Hernández Cifre 2000; Ma and Cheng 2009; Ma and Zhu 2008; Pan and Yang 2008; Sholander 1952].

Chernoff [1969] got an area-width inequality for convex plane curves. Let $\alpha$ be a closed convex curve in the Euclidean plane $\mathbb{R}^{2}$ with area $A$ and width function $w(\theta)$. Then the geometric inequality

$$
A \leq \frac{1}{2} \int_{0}^{\pi / 2} w(\theta) w\left(\theta+\frac{1}{2} \pi\right) d \theta
$$

holds, with equality if and only if $\alpha$ is a circle. To our knowledge, this beautiful inequality has not been generalized yet. One purpose of this note is to make some generalization of the Chernoff inequality. To this end, we introduce for convex

MSC2000: primary 52A38; secondary 52A40.

Keywords: closed convex plane curves, Minkowski's support function, geometric inequality.

Supported in part by National Science Foundation of China number 10871070 and the Shanghai Leading Academic Discipline Project, number B407.

Pan is the corresponding author. 
plane curves a function $w_{k}(\theta)$ for integer $k \geq 2$. This function is defined in (3-1) below and is a generalization of the usual width of a convex curve. Then Chernoff's area-width inequality generalizes as

$$
A \leq \frac{1}{k} \int_{0}^{\pi / k} w_{k}(\theta) w_{k}\left(\theta+\frac{1}{k} \pi\right) d \theta,
$$

with equality if and only if $\alpha$ is a circle. Moreover, we can calculate

$$
\lim _{k \rightarrow \infty} \frac{1}{k} \int_{0}^{\pi / k} w_{k}(\theta) w_{k}\left(\theta+\frac{1}{k} \pi\right) d \theta=\frac{L^{2}}{4 \pi} .
$$

Thus (1-1) and (1-2) give a new proof of the classical isoperimetric inequality $L^{2} \geq 4 \pi A$ with equality if and only if the curve is a circle.

Another purpose here is to give some properties of closed convex curves with $w_{k}(\theta)$ being constant. We will get in Theorem 3.2 an analogue of Barbier's theorem and in Theorem 3.3 we will characterize the support function of such curves. In particular, we pose an open problem that was motivated by the elegant BlaschkeLebesgue theorem.

\section{Preliminaries}

Henceforth suppose without loss of generality that $\alpha$ is a smooth regular positively oriented and closed strictly convex curve in the Euclidean plane $\mathbb{R}^{2}$. Take a point $O$ inside $\alpha$ as the origin of our frame. Let $p$ be the oriented perpendicular distance from $O$ to the tangent at a point on $\alpha$, and $\theta$ the oriented angle from the positive $x_{1}$-axis to this perpendicular ray. Clearly, $p$, as a function of $\theta$, is single-valued and $2 \pi$-periodic. We usually call $p(\theta)$ Minkowski's support function of $\alpha$.

One can check that $\alpha$ can be parametrized in terms of $\theta$ and $p(\theta)$ as

$$
\alpha(\theta)=\left(\alpha_{1}(\theta), \alpha_{2}(\theta)\right)=\left(p(\theta) \cos \theta-p^{\prime}(\theta) \sin \theta, p(\theta) \sin \theta+p^{\prime}(\theta) \cos \theta\right) ;
$$

see for instance [Hsiung 1981]. The curvature $\kappa$ of $\alpha$ can be calculated according to $\kappa(\theta)=d \theta / d s=1 /\left(p(\theta)+p^{\prime \prime}(\theta)\right)>0$. Denote by $L$ and $A$ the length of $\alpha$ and the area it bounds. Then one can get

$$
\begin{aligned}
L=\int_{\alpha} d s=\int_{0}^{2 \pi} \rho(\theta) d \theta=\int_{0}^{2 \pi} p(\theta) d \theta & \\
A=\frac{1}{2} \int_{\alpha} p(\theta) d s & =\frac{1}{2} \int_{0}^{2 \pi} p(\theta)\left(p(\theta)+p^{\prime \prime}(\theta)\right) d \theta \\
& =\frac{1}{2} \int_{0}^{2 \pi}\left(p^{2}(\theta)-p^{\prime 2}(\theta)\right) d \theta .
\end{aligned}
$$

These are known as Cauchy's formula and Blaschke's formula, respectively. 
Since the support function of a given convex curve $\alpha$ is always continuous, bounded and $2 \pi$-periodic, it has a Fourier series of the form

$$
p(\theta)=\frac{1}{2} a_{0}+\sum_{n=1}^{\infty}\left(a_{n} \cos n \theta+b_{n} \sin n \theta\right),
$$

where

$$
\begin{aligned}
a_{0}=\frac{1}{\pi} \int_{0}^{2 \pi} p(\theta) d \theta, \quad a_{n} & =\frac{1}{\pi} \int_{0}^{2 \pi} p(\theta) \cos n \theta d \theta, \\
b_{n} & =\frac{1}{\pi} \int_{0}^{2 \pi} p(\theta) \sin n \theta d \theta \quad \text { for } n \geq 1 .
\end{aligned}
$$

We wish to express $L$ and $A$ in terms of the Fourier coefficients of $p(\theta)$. From (2-1) and the first equation of (2-4) one easily sees that $L=\pi a_{0}$. Then differentiating (2-3) with respect to $\theta$ gives us $p^{\prime}(\theta)=\sum_{n=1}^{\infty} n\left(-a_{n} \sin n \theta+b_{n} \cos n \theta\right)$. By the Parseval equality and (2-2), we get

$$
A=\frac{1}{4} \pi a_{0}^{2}+\frac{1}{2} \pi \sum_{n=2}^{\infty}\left(1-n^{2}\right)\left(a_{n}^{2}+b_{n}^{2}\right),
$$

The width $w(\theta)$ of $\alpha$ in direction $\boldsymbol{u}(\theta)=(\cos \theta, \sin \theta)$ is defined to be the distance between two tangents to $\alpha$ perpendicular to $\boldsymbol{u}(\theta)$. It is clear that

$$
w(\theta)=p(\theta)+p(\theta+\pi) .
$$

The closed convex curve $\alpha$ is said to be of constant width if its width in any direction is a positive constant $w_{0}$, and in this case, the constant $w_{0}$ is called the width of $\alpha$. If $\alpha$ is a constant width curve with width $w_{0}$, then $p(\theta)+p(\theta+\pi)=w_{0}$ for any $\theta \in[0,2 \pi]$.

It is obvious that a circle is a constant width curve. There are, however,- many other noncircular curves of constant width; see for example [Burke 1966; Hsiung 1981; Rabinowitz 1997]. Among them, the most famous example is the Reuleaux triangle, which has been used in the design of piston for the Wankel engine. A famous result about constant width curves due to Barbier [1860] states that all closed convex curves of constant width $w_{0}$ have the same perimeter $\pi w_{0}$. Another elegant result is the Blaschke-Lebesgue theorem, which says that among all closed convex curves with constant width $w_{0}$, the Reuleaux triangles of the same constant width have the smallest area.

Our Theorem 3.2 bears analogy to Barbier's theorem. The open problem posed in the next section is motivated by the Blaschke-Lebesgue theorem first proved by Blaschke [1915] and Lebesgue [1914; 1921]. Harrell [2002] gives a new proof of 
this theorem and some historic remarks on it. The higher dimensional BlaschkeLebesgue problem appears to be very difficult to solve and remains open. For partial results, see [Anciaux and Georgiou 2009; Anciaux and Guilfoyle 2010] and the literature therein.

\section{Main results}

For an integer $k \geq 2$, we introduce for a convex curve $\alpha$ a function $w_{k}(\theta)$ by

$$
w_{k}(\theta)=p(\theta)+p(\theta+2 \pi / k)+\cdots+p(\theta+(2(k-1) \pi) / k) .
$$

Since

$$
\begin{aligned}
1+\cos (2 \pi / k)+\cdots+\cos (2(k-1) \pi / k) & =0, \\
\sin (2 \pi / k)+\cdots+\sin (2(k-1) \pi / k) & =0,
\end{aligned}
$$

the function $w_{k}(\theta)$ is independent of the choice of the origin $O$ (inside $\alpha$ ) and thus is well-defined. It is clear that $w_{k}(\theta)$ is a periodic function with period $2 \pi / k$.

If $k=2, w_{2}(\theta)$ is the usual width (see (2-6)) of a curve, that is, our $w_{k}(\theta)$ is a generalization of the usual width function $w(\theta)$. In this case, Chernoff [1969] got a nice area-width inequality. For general $k$, we can generalize:

Theorem 3.1. Let $\alpha$ be a closed convex plane curve, bounding a region of area A. Then

$$
A \leq \frac{1}{k} \int_{0}^{\pi / k} w_{k}(\theta) w_{k}(\theta+\pi / k) d \theta,
$$

where the equality holds if and only if $\alpha$ is a circle.

Proof. The proof is divided into two steps.

Step 1. We first show that

$$
\int_{0}^{\pi / k} w_{k}(\theta) w_{k}(\theta+\pi / k) d \theta=\frac{1}{2} \sum_{m=1}^{k} \int_{0}^{2 \pi} p(\theta) p(\theta+(2 m-1) \pi / k) d \theta .
$$

To see this, write

$$
a_{i j}=\int_{0}^{\pi / k} p\left(\theta+\frac{(2 i-1) \pi}{k}\right) p\left(\theta+\frac{2(j-1) \pi}{k}\right) d \theta \quad \text { for } i, j=1,2, \ldots, 2 k .
$$

Then

$$
\begin{aligned}
& \int_{0}^{\pi / k} w_{k}(\theta) w_{k}\left(\theta+\frac{\pi}{k}\right) d \theta \\
= & \int_{0}^{\pi / k}\left(p(\theta)+p\left(\theta+\frac{2 \pi}{k}\right)+\cdots+p\left(\theta+\frac{2(k-1) \pi}{k}\right)\right) \\
& \quad\left(p\left(\theta+\frac{\pi}{k}\right)+p\left(\theta+\frac{3 \pi}{k}\right)+\cdots+p\left(\theta+\frac{(2 k-1) \pi}{k}\right)\right) d \theta=\sum_{i, j=1}^{k} a_{i j} .
\end{aligned}
$$


Since $p$ is a $2 \pi$-periodic function, we get

$$
a_{i+k, j}=a_{i j}=a_{i, j+k} \quad \text { for } i, j=1,2, \ldots, k .
$$

Now, we claim that

$$
\sum_{m=1}^{k} \sum_{l=1}^{k} a_{m+l-1, l}=\sum_{m=1}^{k} \sum_{l=1}^{k} a_{l, m+l}=\sum_{i, j=1}^{k} a_{i j} .
$$

The sum at left can be treated as

$\sum_{l=1}^{k}\left(\sum_{m=1}^{k-l+1} a_{m+l-1, l}+\sum_{m=k-l+2}^{k} a_{m+l-k-1, l}\right)=\sum_{l=1}^{k}\left(\sum_{i=l}^{k} a_{i l}+\sum_{i=1}^{l-1} a_{i l}\right)=\sum_{l=1}^{k} \sum_{i=1}^{k} a_{i l}$,

while the middle sum becomes

$$
\sum_{l=1}^{k}\left(\sum_{m=1}^{k-l} a_{l, m+l}+\sum_{m=k-l+1}^{k} a_{l, m+l-k}\right)=\sum_{l=1}^{k}\left(\sum_{i=l+1}^{k} a_{l i}+\sum_{i=1}^{l} a_{l i}\right)=\sum_{l=1}^{k} \sum_{i=1}^{k} a_{l i} .
$$

Thus, we get

$$
\int_{0}^{\pi / k} w_{k}(\theta) w_{k}(\theta+\pi / k) d \theta=\sum_{i, j=1}^{k} a_{i j}=\frac{1}{2} \sum_{m=1}^{k}\left(\sum_{l=1}^{k}\left(a_{m+l-1, l}+a_{l, m+l}\right)\right) .
$$

Next, we shall show that, for $1 \leq m \leq k$,

$$
\sum_{l=1}^{k}\left(a_{m+l-1, l}+a_{l, m+l}\right)=\int_{0}^{2 \pi} p(\theta) p(\theta+(2 m-1) \pi / k) d \theta .
$$

In fact,

$$
\begin{aligned}
\text { left side of (3-7) }= & \sum_{l=1}^{k}\left(\int_{0}^{\pi / k} p\left(\theta+\frac{2(m+l-1)-1}{k} \pi\right) p\left(\theta+\frac{2(l-1)}{k} \pi\right) d \theta\right. \\
& \left.\quad+\int_{0}^{\pi / k} p\left(\theta+\frac{2 l-1}{k} \pi\right) p\left(\theta+\frac{2(m+l-1)}{k} \pi\right) d \theta\right) \\
= & \sum_{l=1}^{k}\left(\int_{2(l-1) \pi / k}^{(2 l-1) \pi / k} p(\theta) p\left(\theta+\frac{(2 m-1) \pi}{k}\right) d \theta\right. \\
& \left.\quad+\int_{(2 l-1) \pi / k}^{2 l \pi / k} p(\theta) p\left(\theta+\frac{(2 m-1) \pi}{k}\right) d \theta\right) \\
= & \int_{0}^{2 \pi} p(\theta) p\left(\theta+\frac{(2 m-1) \pi}{k}\right) d \theta .
\end{aligned}
$$

Now, combining (3-3)-(3-7) yields (3-2). 
Step 2. After some calculations, we get, for $1 \leq m \leq k$,

$$
\int_{0}^{2 \pi} p(\theta) p\left(\theta+\frac{(2 m-1) \pi}{k}\right) d \theta=\frac{1}{2} \pi a_{0}^{2}+\pi \sum_{n=1}^{\infty}\left(a_{n}^{2}+b_{n}^{2}\right) \cos \frac{n(2 m-1) \pi}{k}
$$

For any integer $n$ not a multiple of $k$, we have

$$
\begin{aligned}
\sum_{m=1}^{k} \cos \frac{n(2 m-1) \pi}{k} & =\frac{1}{\sin (n \pi / k)} \sum_{m=1}^{k}\left(\cos \frac{n(2 m-1) \pi}{k} \sin \frac{n \pi}{k}\right) \\
& =\frac{\sin 2 n \pi}{2 \sin (n \pi / k)}=0 .
\end{aligned}
$$

It follows from (3-2), (3-8), (3-9) and (2-5) that

$$
\begin{aligned}
\frac{1}{k} \int_{0}^{\pi / k} w_{k}(\theta) w_{k}\left(\theta+\frac{\pi}{k}\right) d \theta=\frac{1}{2 k} \sum_{m=1}^{k} \int_{0}^{2 \pi} p(\theta) p\left(\theta+\frac{(2 m-1) \pi}{k}\right) d \theta \\
=\frac{1}{4} \pi a_{0}^{2}+\frac{\pi}{2} \sum_{n=1}^{\infty}\left(a_{n}^{2}+b_{n}^{2}\right) \frac{1}{k} \sum_{m=1}^{k} \cos \frac{n(2 m-1) \pi}{k} \\
=\frac{1}{4} \pi a_{0}^{2}+\frac{\pi}{2} \sum_{l=1}^{\infty}\left(a_{k l}^{2}+b_{k l}^{2}\right) \frac{1}{k} \sum_{m=1}^{k} \cos (l(2 m-1) \pi) \\
=\frac{1}{4} \pi a_{0}^{2}+\frac{\pi}{2} \sum_{l=1}^{\infty}(-1)^{l}\left(a_{k l}^{2}+b_{k l}^{2}\right) \\
=A+\frac{\pi}{2}\left(\sum_{n=2}^{\infty}\left(a_{n}^{2}+b_{n}^{2}\right)\left(n^{2}-1\right)+\sum_{l=1}^{\infty}(-1)^{l}\left(a_{k l}^{2}+b_{k l}^{2}\right)\right) \\
\geq A .
\end{aligned}
$$

The equality holds if and only if $a_{n}=b_{n}=0$ for all $n \geq 2$, that is, when the curve is a circle.

From the continuity of $p(\theta)$, it is easy to see that, for all $\theta_{k} \in[0,2 \pi / k]$,

$$
\lim _{k \rightarrow \infty} \frac{2 \pi}{k} w_{k}\left(\theta_{k}\right)=\lim _{k \rightarrow \infty} \frac{2 \pi}{k} \sum_{m=1}^{k} p\left(\theta_{k}+\frac{2 m \pi}{k}\right)=\int_{0}^{2 \pi} p(\theta) d \theta .
$$

Moreover, for any $k \in \mathbb{N}$, there exists a $\xi_{k} \in[0, \pi / k]$ such that

$$
\frac{1}{k} \int_{0}^{\pi / k} w_{k}(\theta) w_{k}(\theta+\pi / k) d \theta=\frac{\pi}{k^{2}} w_{k}\left(\xi_{k}\right) w_{k}\left(\xi_{k}+\pi / k\right) .
$$


Since $\xi_{k} \in[0, \pi / k] \subset[0,2 \pi / k]$, we have $\xi_{k}+\pi / k \in[0,2 \pi / k]$. Thus, we obtain

$$
\begin{aligned}
\lim _{k \rightarrow \infty} \frac{1}{k} \int_{0}^{\pi / k} w_{k}(\theta) w_{k}(\theta+\pi / k) d \theta & =\lim _{k \rightarrow \infty} \frac{\pi}{k^{2}} w_{k}\left(\xi_{k}\right) w_{k}\left(\xi_{k}+\pi / k\right) \\
& =\frac{1}{4 \pi}\left(\int_{0}^{2 \pi} p(\theta) d \theta\right)^{2}=\frac{L^{2}}{4 \pi} .
\end{aligned}
$$

which with (3-10) gives us a new proof of the classical isoperimetric inequality.

We can also get the following generalization of Barbier's theorem.

Theorem 3.2. All convex curves for $w_{k}(\theta)$ is equal to a constant $\Lambda$ have the same length $L=(2 \pi / k) \Lambda$.

Proof. It is easy to see from (2-1) that $L=\int_{0}^{2 \pi / k} w_{k}(\theta) d \theta=(2 \pi / k) \Lambda$.

Among all curves with the same length $L$, circles have the greatest area. For constant width curves, the Blaschke-Lebesgue theorem claims that the Reuleaux triangles have the least area.

Question. Among all closed convex curves with $w_{k}(\theta)$ equal to a fixed consant $\Lambda$, which has the least possible area?

Theorem 3.3. Suppose $\alpha$ is a closed convex plane curve with $w_{k}(\theta)$ equal to a constant $\Lambda$. Then the Fourier expansion of the support function $p(\theta)$ of $\alpha$ is of the form

$$
p(\theta)=\frac{1}{2} a_{0}+\sum_{n=1, n \neq m k}^{\infty}\left(a_{n} \cos n \theta+b_{n} \sin n \theta\right),
$$

where $a_{0}=(1 / \pi) \int_{0}^{2 \pi} p(\theta) d \theta=L / \pi=2 \Lambda / k$ and $m \in \mathbb{N}$.

Proof. In terms of the Fourier expansion of the support function $p(\theta)$ of $\alpha$,

$$
\begin{gathered}
w_{k}(\theta)=\frac{1}{2} k a_{0}+\sum_{n=1}^{\infty}\left(a_{n} \cos n \theta+b_{n} \sin n \theta+a_{n} \cos \left(n \theta+\frac{2 n \pi}{k}\right)+b_{n} \sin \left(n \theta+\frac{2 n \pi}{k}\right)\right. \\
\left.+\cdots+a_{n} \cos \left(n \theta+\frac{2 n(k-1) \pi}{k}\right)+b_{n} \sin \left(n \theta+\frac{2 n(k-1) \pi}{k}\right)\right) \\
=\frac{1}{2} k a_{0}+\sum_{n=1}^{\infty}\left(\left(a_{n} \cos n \theta+b_{n} \sin n \theta\right)\left(1+\cos \frac{2 n \pi}{k}+\cdots+\cos \frac{2 n(k-1) \pi}{k}\right)\right. \\
\left.+\left(b_{n} \cos n \theta-a_{n} \sin n \theta\right)\left(\sin \frac{2 n \pi}{k}+\sin \frac{4 n \pi}{k}+\cdots+\sin \frac{2 n(k-1) \pi}{k}\right)\right) \\
=\frac{1}{2} k a_{0}+k \sum_{n=1}^{\infty}\left(a_{k n} \cos (k n \theta)+b_{k n} \sin (k n \theta)\right) .
\end{gathered}
$$

If $w_{k}(\theta)=\Lambda$, then one gets $a_{0}=(2 / k) \Lambda$ and $a_{k n}=0=b_{k n}$. 


\section{Acknowledgements}

We are grateful to the anonymous referee for his or her careful reading of the original manuscript and giving us some invaluable comments.

\section{References}

[Anciaux and Georgiou 2009] H. Anciaux and N. Georgiou, "The Blaschke-Lebesgue problem for constant width bodies of revolution", preprint, version 1, 2009. arXiv 0903.4284v1

[Anciaux and Guilfoyle 2010] H. Anciaux and B. Guilfoyle, "On the three-dimensional BlaschkeLebesgue problem", preprint, version 2, 2010. arXiv 0906.3217v2

[Ball 1991] K. Ball, "Volume ratios and a reverse isoperimetric inequality", J. London Math. Soc. (2) 44:2 (1991), 351-359. MR 92j:52013 Zbl 0694.46010

[Barbier 1860] A. Barbier, "Note sur le problème de l'aiguille le et jeu du joint couvert", J. Math. Pure Appl (2) 5 (1860), 273-286.

[Blaschke 1915] W. Blaschke, "Konvexe Bereiche gegebener konstanter Breite und kleinsten Inhalts", Math. Ann. 76:4 (1915), 504-513. MR 1511839 JFM 45.0731.04

[Blaschke 1956] W. Blaschke, Kreis und Kugel, 2nd ed., de Gruyter, Berlin, 1956. MR 17,1123d Zbl 0070.17501

[Bonnesen 1929] T. Bonnesen, Les problèmes des isopérimètres et des isépiphanes, Gauthier-Villars, Paris, 1929. JFM 55.0431.08

[Bonnesen and Fenchel 1934] T. Bonnesen and W. Fenchel, Theorie der konvexen Körper, Ergebnisse der Mathematik und ihrer Grenzgebiete (3) 1, Springer, 1934. MR 51 \#8954 Zbl 0008.07708 JFM 60.0673.01

[Burago and Zalgaller 1988] Y. D. Burago and V. A. Zalgaller, Geometric inequalities, Grundlehren der Mathematischen Wissenschaften 285, Springer, Berlin, 1988. MR 89b:52020 Zbl 0633.53002

[Burke 1966] J. F. Burke, “A curve of constant diameter”, Math. Mag. 39 (1966), 84-85. MR 33 \#1794 Zbl 0136.18903

[Chernoff 1969] P. R. Chernoff, "An area-width inequality for convex curves", Amer. Math. Monthly 76 (1969), 34-35. MR 38 \#3769 Zbl 0175.19501

[Gage 1983] M. E. Gage, "An isoperimetric inequality with applications to curve shortening", Duke Math. J. 50:4 (1983), 1225-1229. MR 85d:52007 Zbl 0534.52008

[Green and Osher 1999] M. Green and S. Osher, "Steiner polynomials, Wulff flows, and some new isoperimetric inequalities for convex plane curves", Asian J. Math. 3:3 (1999), 659-676. MR 2001j:53089 Zbl 0969.53040

[Harrell 2002] E. M. Harrell, II, "A direct proof of a theorem of Blaschke and Lebesgue", J. Geom. Anal. 12:1 (2002), 81-88. MR 2002k:52009 Zbl 1044.52001

[Hernández Cifre 2000] M. A. Hernández Cifre, "Is there a planar convex set with given width, diameter, and inradius?", Amer. Math. Monthly 107:10 (2000), 893-900. MR 2001j:52002 Zbl 0983.52002

[Hsiung 1981] C. C. Hsiung, A first course in differential geometry, Wiley, New York, 1981. MR 83c:53001 Zbl 0458.53001

[Lebesgue 1914] H. Lebesgue, "Sur le problème des isopérimètres et sur les domaines de largeur constante”, Bull. Soc. Math. France C. R. 7 (1914), 72-76. 
[Lebesgue 1921] H. Lebesgue, "Sur quelques questions des minimums, relatives aux courbes orbiformes, et sur leurs rapports avec le calcul de variations", J. Math. Pure Appl. 8 (1921), 67-96. JFM 48.0838.01

[Ma and Cheng 2009] L. Ma and L. Cheng, "A non-local area preserving curve flow", preprint, 2009. arXiv 0907.1430

[Ma and Zhu 2008] L. Ma and A. Zhu, "On a length preserving curve flow", preprint, 2008. arXiv 0811.2083

[Osserman 1978] R. Osserman, "The isoperimetric inequality”, Bull. Amer. Math. Soc. 84:6 (1978), 1182-1238. MR 58 \#18161 Zbl 0411.52006

[Osserman 1979] R. Osserman, "Bonnesen-style isoperimetric inequalities", Amer. Math. Monthly 86:1 (1979), 1-29. MR 80h:52013 Zbl 0404.52012

[Pan and Yang 2008] S. Pan and J. Yang, "On a non-local perimeter-preserving curve evolution problem for convex plane curves”, Manuscripta Math. 127:4 (2008), 469-484. MR 2010h:53099 Zbl 1169.35033

[Pan and Zhang 2007] S. Pan and H. Zhang, "A reverse isoperimetric inequality for convex plane curves”, Beiträge Algebra Geom. 48:1 (2007), 303-308. MR 2008c:52011 Zbl 1121.52023

[Rabinowitz 1997] S. Rabinowitz, "A polynomial curve of constant width", Missouri J. Math. Sci. 9:1 (1997), 23-27. MR 98d:52002 Zbl 1097.52501

[Schneider 1993] R. Schneider, Convex bodies: the Brunn-Minkowski theory, Encyclopedia of Mathematics and its Applications 44, Cambridge University Press, 1993. MR 94d:52007 Zbl 0798.52001

[Sholander 1952] M. Sholander, "On certain minimum problems in the theory of convex curves", Trans. Amer. Math. Soc. 73 (1952), 139-173. MR 14,787d Zbl 0047.15901

Received September 10, 2009. Revised November 13, 2009.

KE OU

DEPARTMENT OF MATHEMATICS, EAST CHINA NORMAL UNIVERSITY

SHANGHAI 200241

CHINA

justinok1311@163.com

SHENGLIANG PAN

DEPARTMENT OF MATHEMATICS

TONGJI UNIVERSITY

SHANGHAI 200092

CHINA

slpan@ tongji.edu.cn 


\title{
PACIFIC JOURNAL OF MATHEMATICS
}

\author{
http://www.pjmath.org \\ Founded in 1951 by \\ E. F. Beckenbach (1906-1982) and F. Wolf (1904-1989)
}

\section{EDITORS}

V. S. Varadarajan (Managing Editor)

Department of Mathematics

University of California

Los Angeles, CA 90095-1555

pacific@math.ucla.edu

Vyjayanthi Chari

Department of Mathematics

University of California

Riverside, CA 92521-0135

chari@math.ucr.edu

Robert Finn

Department of Mathematics Stanford University

Stanford, CA 94305-2125

finn@math.stanford.edu

Kefeng Liu

Department of Mathematics

University of California

Los Angeles, CA 90095-1555

liu@math.ucla.edu
Darren Long

Department of Mathematics

University of California

Santa Barbara, CA 93106-3080

long@math.ucsb.edu

Jiang-Hua Lu

Department of Mathematics

The University of Hong Kong

Pokfulam Rd., Hong Kong jhlu@maths.hku.hk

Alexander Merkurjev

Department of Mathematics

University of California

Los Angeles, CA 90095-1555

merkurev@math.ucla.edu
Sorin Popa

Department of Mathematics University of California

Los Angeles, CA 90095-1555 popa@math.ucla.edu

Jie Qing

Department of Mathematics

University of California

Santa Cruz, CA 95064

qing@cats.ucsc.edu

Jonathan Rogawski

Department of Mathematics

University of California

Los Angeles, CA 90095-1555

jonr@math.ucla.edu

\section{PRODUCTION}

pacific@math.berkeley.edu

\begin{abstract}
Silvio Levy, Scientific Editor Matthew Cargo, Senior Production Editor
\end{abstract}
ACADEMIA SINICA, TAIPEI

CALIFORNIA INST. OF TECHNOLOGY

INST. DE MATEMÁTICA PURA E APLICADA

KEIO UNIVERSITY

MATH. SCIENCES RESEARCH INSTITUTE

NEW MEXICO STATE UNIV.

OREGON STATE UNIV.

\section{SUPPORTING INSTITUTIONS}

STANFORD UNIVERSITY
UNIV. OF BRITISH COLUMBIA
UNIV. OF CALIFORNIA, BERKELEY
UNIV. OF CALIFORNIA, DAVIS
UNIV. OF CALIFORNIA, LOS ANGELES
UNIV. OF CALIFORNIA, RIVERSIDE
UNIV. OF CALIFORNIA, SAN DIEGO
UNIV. OF CALIF., SANTA BARBARA

UNIV. OF CALIF., SANTA CRUZ

UNIV. OF MONTANA

UNIV. OF OREGON

UNIV. OF SOUTHERN CALIFORNIA

UNIV. OF UTAH

UNIV. OF WASHINGTON

WASHINGTON STATE UNIVERSITY

These supporting institutions contribute to the cost of publication of this Journal, but they are not owners or publishers and have no responsibility for its contents or policies.

See inside back cover or www.pjmath.org for submission instructions.

The subscription price for 2010 is US \$420/year for the electronic version, and \$485/year for print and electronic.

Subscriptions, requests for back issues from the last three years and changes of subscribers address should be sent to Pacific Journal of Mathematics, P.O. Box 4163, Berkeley, CA 94704-0163, U.S.A. Prior back issues are obtainable from Periodicals Service Company, 11 Main Street, Germantown, NY 12526-5635. The Pacific Journal of Mathematics is indexed by Mathematical Reviews, Zentralblatt MATH, PASCAL CNRS Index, Referativnyi Zhurnal, Current Mathematical Publications and the Science Citation Index.

The Pacific Journal of Mathematics (ISSN 0030-8730) at the University of California, c/o Department of Mathematics, 969 Evans Hall, Berkeley, CA 94720-3840, is published monthly except July and August. Periodical rate postage paid at Berkeley, CA 94704, and additional mailing offices. POSTMASTER: send address changes to Pacific Journal of Mathematics, P.O. Box 4163, Berkeley, CA 94704-0163.

PJM peer review and production are managed by EditFLOW ${ }^{\mathrm{TM}}$ from Mathematical Sciences Publishers.

PUBLISHED BY PACIFIC JOURNAL OF MATHEMATICS

at the University of California, Berkeley 94720-3840

A NON-PROFIT CORPORATION

Typeset in LATEX

Copyright $(\mathrm{C} 2010$ by Pacific Journal of Mathematics 


\section{PACIFIC JOURNAL OF MATHEMATICS}

Volume $248 \quad$ No. $2 \quad$ December 2010

Topological description of Riemannian foliations with dense leaves

Jesús A. ÁlVAREZ LóPEZ and Alberto CANDEL

The nonexistence of quasi-Einstein metrics

JEFFREY S. CASE

Twisted symmetric group actions

AKINARI HoSHI and MING-CHANG KANG

Optimal transportation and monotonic quantities on evolving manifolds

305

HONG HUANG

Hopf structures on the Hopf quiver $Q(\langle g\rangle, g)$

HuA-Lin HuANG, YU Ye and QING ZHaO

Minimal surfaces in $S^{3}$ foliated by circles

Nikolai KuteV and VelichKa Milousheva

Prealternative algebras and prealternative bialgebras

XIANG Ni and CHENGMING BAI

Some remarks about closed convex curves

Ke OU and Shengliang PAN

Orbit correspondences for real reductive dual pairs

SHU-YEN PAN

Graphs of bounded degree and the $p$-harmonic boundary

Michael J. Puls

Invariance of the BFV complex

FLORIAN SCHÄTZ

Some elliptic PDEs on Riemannian manifolds with boundary

YANNICK SIRE and ENRICO VALDINOCI

Representations of Lie superalgebras in prime characteristic, III 